Cahiers

Balkaniques

\section{Cahiers balkaniques}

Hors-série | 2015

Les élites grecques modernes, $\mathrm{XVIII} \mathrm{e}^{\mathrm{X}} \mathrm{XX}^{\mathrm{e}}$ siècles : identités, modes d'action, représentations

\title{
L'« élite » surréaliste grecque comme précurseur des ouvertures cruciales de l'esprit
}

The Greek Surrealist "Elite" as a Precursor to Crucial Openings of The Mind

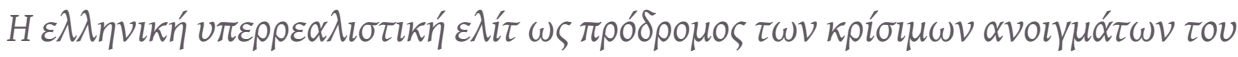

$\pi v \varepsilon v ́ \mu \alpha \tau o \varsigma$

\section{Diamanti Anagnostopoulou}

\section{OpenEdition}

\section{Journals}

Édition électronique

URL : https://journals.openedition.org/ceb/5739

DOI : 10.4000/ceb.5739

ISSN : 2261-4184

Éditeur

INALCO

\section{Édition imprimée}

Date de publication : 1 mars 2015

ISBN : 978-2-85831-224-5

ISSN : 0290-7402

\section{Référence électronique}

Diamanti Anagnostopoulou, «L'« élite » surréaliste grecque comme précurseur des ouvertures cruciales de l'esprit », Cahiers balkaniques [En ligne], Hors-série | 2015, mis en ligne le 17 novembre 2015, consulté le 06 juillet 2021. URL : http://journals.openedition.org/ceb/5739; DOI : https://doi.org/ 10.4000/ceb.5739

Ce document a été généré automatiquement le 6 juillet 2021.

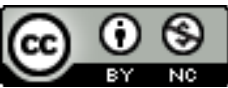

Cahiers balkaniques est mis à disposition selon les termes de la Licence Creative Commons Attribution - Pas d'Utilisation Commerciale 4.0 International. 


\title{
L'« élite » surréaliste grecque comme précurseur des ouvertures cruciales de l'esprit
}

\author{
The Greek Surrealist "Elite" as a Precursor to Crucial Openings of The Mind

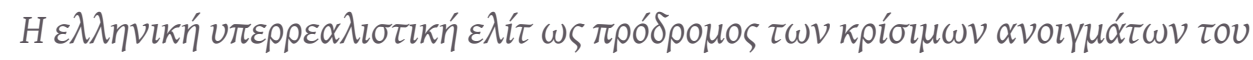 \\ $\pi v \varepsilon v ́ \mu \alpha \tau o s$
}

Diamanti Anagnostopoulou

1 La conception surréaliste met en scène poétique les forces agissantes du désir, la quête de la connaissance de l'Autre et la conciliation du champ intérieur et du champ extérieur. Dans l'écriture surréaliste, le mouvement du désir résulte de deux altérités : l'interdit venant des autres et la force de la pulsion venant de l'intérieur. Dans leur démarche de transgression des contraintes extérieures et intérieures, les surréalistes tentent de réinventer une certaine orientation de l'existence, ainsi qu'une recomposition du système de valeurs morales et intellectuelles. À sa manière, le surréalisme s'est efforcé de concilier un entre-deux réalités (intérieure/psychique et extérieure/objective) et un entre-deux sexes, et l'écriture s'est affirmée comme un moyen d'explorer et de penser ces entre-deux. À travers eux, les surréalistes abordent des thèmes tels que le désir, l'érotisme et la quête de l'amour, la résistance à l'hypocrisie, l'invention poétique et la force de l'imaginaire, la liberté politique et sociale. Ces thèmes sont redéfinis à travers le discours poétique qui devient un champ de communication et de découverte. Il s'agit ainsi d'une " poétique qui bouleverse la pensée et couvre l'ensemble des activités humaines sur le mode de la réflexivité, de la

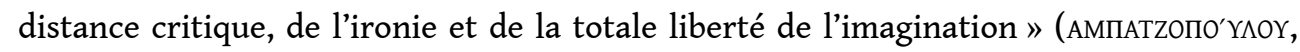
2001, 33).

2 En Grèce, le surréalisme apparaît dans un contexte historique difficile et dans une période de grands changements dans le domaine de la littérature. Affilié au mouvement moderniste, il en constitue une version spécifique qui inscrit le désir au centre du langage et l'amour au centre de la vie de l'homme. Il introduit la contestation 
généralisée des valeurs établies et des conventions et tente de tisser, à travers le discours poétique, un réseau d'éléments nouveaux qui bouleversent la tradition littéraire et le formalisme linguistique. La tradition n'est plus une réalité immuable et

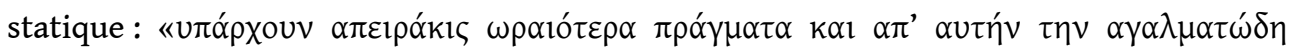

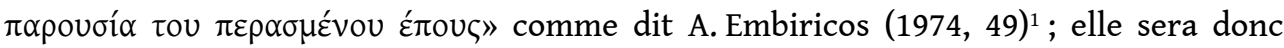

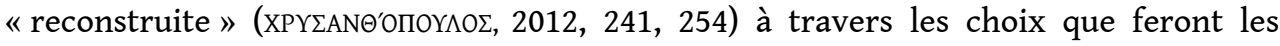
surréalistes grecs en matière d'écrivains, de textes et de courants artistiques.

3 Nous allons nous concentrer sur cette période fertile de l'entre-deux-guerres pendant laquelle sont publiés les textes écrits par Embiricos et par Engonopoulos jusqu'en 1945, et dans laquelle émerge la réception positive ou négative de leur œuvre par le public de l'époque et par leurs pairs. Nous tenterons d'examiner dans quelle mesure ces deux représentants du surréalisme ont réussi à assumer le rôle d'élite dans la formation d'une nouvelle identité culturelle et littéraire de leur temps, la façon dont ils se sont mesurés aux interdits, aux conflits et à leur transgression et nous explorerons les représentations de cette rupture avec le passé dans leur œuvre.

\section{Le surréalisme dans l'entre-deux-guerres}

Le surréalisme grec suscite d'emblée la réprobation de la gauche qui le perçoit comme une illustration du snobisme de la bourgeoisie décadente ( $\left.\Delta^{\prime} \mathrm{E} \Lambda \mathrm{IO \Sigma}, 1935,329-330\right)$ et l'accuse de dissoudre la rime et d'une subjectivité absolue qui coupe l'art du monde extérieur et l'abandonne au chaos et à l'incohérence ${ }^{2}$. Dans les autres cas, il rencontre l'indifférence, l'ironie ${ }^{3}$ et un silence prudent. Cette réticence provient :

5 a) de l'inexistence de textes grecs définissant le surréalisme ainsi que de textes théoriques étrangers traduits en grec, c'est-à-dire de l'absence des fondements théoriques nécessaires à la production d'un discours critique substantiel ;

b) de la réception restreinte de la théorie freudienne et de ses concepts fondamentaux ;

c) de la transgression du canon littéraire par les surréalistes ;

d) de l'attitude négative de Séféris $(1981,85,485,476)$ qui imposa, comme le dit par la suite eLYTIs $(1982,378-394)$ son point de vue dans le débat littéraire qui s'est déroulé

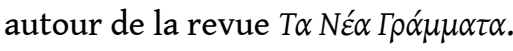

6 Le « caractère impersonnel » de l'inspiration ${ }^{4}$ et l'écriture automatique suscitaient chez Séféris la plus forte méfiance à l'égard du surréalisme. La même année où Séféris prend

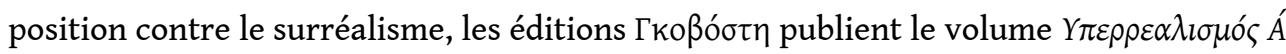
(1938) qui contient, outre des traductions de textes de surréalistes français par leurs pairs grecs, une liste d'œuvres de surréalistes grecs. Ce volume est important, car il s'agit de leur première apparition collective (АРГҮР'IOY, 1983, 13-14).

7 A. Embiricos inaugure sa poétique et le surréalisme en Grèce par des poèmes d'écriture automatique. S'il abandonne partiellement cette pratique dans son recueil suivant -

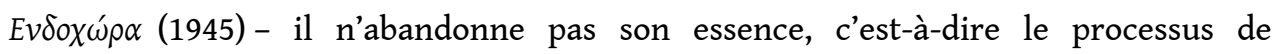
substitution métaphorique soutenu par l'élément a-logique. Pour les surréalistes, l'écriture automatique fonctionne comme un tremplin d'inspiration, comme exaltation des signes psychiques, abolition des distinctions entre écrit littéraire et non littéraire, suppression des frontières entre imaginaire et réel, entre subjectif et objectif. Dans le premier Manifeste (1924), A. Breton définit le surréalisme comme "automatisme psychique pur par lequel on se propose d'exprimer, soit verbalement, soit par écrit [...] 
le fonctionnement réel de la pensée " $(1979,37)$. L'identification du surréalisme à l'automatisme dans le premier Manifeste annonce clairement le refus intérieur de la suprématie de la logique et du contrôle. L'automatisme s'entend comme un processus libérateur qui favorise l'expression pure de la pensée, qui permet à la conscience d'atteindre un niveau supérieur à celui des activités quotidiennes régies par la pensée rationaliste et ses contraintes. Manifestation de la pensée et de l'esprit, l'automatisme, tel qu'il se décline à travers les termes de Breton " coulée verbale ", " pensée parlée " (p. 34), « dictée ", « écriture de la pensée » (p. 35), et d'Aragon "matière mentale ", concerne le langage de l'inconscient, le Ça, qui parle à l'intérieur de tout sujet. Ce langage «se formait dans cette zone de l'activité psychique d'où viennent les pulsions instinctives, les images primordiales, les rêves, ce qui supposait que l'inconscient se manifeste spontanément comme langage, qu'il est une structure linguistique, un langage potentiel qui se réalise dès que les clôtures qui le retenaient prisonnier sont supprimées » (BRÉCHON, 1971, 34).

8 Pour les surréalistes, les mots, à travers leur émergence fortuite, viennent signifier et rendre visible ce qui était occulté. Ils jouent avec l'inconnu comme enjeu. Le hasard révèle à la pensée et au monde ce qui ne se manifeste qu'à travers la rencontre. L'écriture automatique apparaît comme la preuve de l'infaillibilité de l'improbable. À travers elle, le sujet créateur tente de se débarrasser du poids de la représentation, de

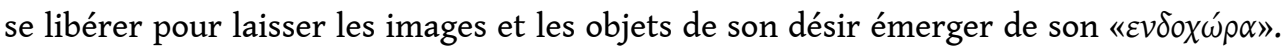
En accord avec ses références freudiennes, A. Embiricos pense que le langage est ancré dans la sexualité et que la civilisation a progressivement condamné la source du désir ainsi que les jeux inhérents au verbe. Pour accéder de nouveau à la source, il faut donc observer une véritable ascèse mentale d'ordre éthique et esthétique. Il recourt ainsi à l'écriture automatique et, par la suite, à des principes d'écriture automatique pour articuler le langage de l'amour et de la poésie, car dans son œuvre, l'érotique revêt toujours l'esthétique. L'automatisme n'est pas le royaume de l'informe. Malgré leurs aspects irrationnels et paradoxaux, ses productions sont des constructions signifiantes « remarquablement coordonnées » soulignait Breton dans les Champs Magnétiques. Les associations paradoxales entre mots, images, objets, personnages et événements, déploient une métaphore continue employée comme une "fenêtre dont il faut savoir sur quoi elle donne et si là où elle s'ouvre, la vue est belle " (BRETON)5. Cette métaphore porte en elle des moments du merveilleux et elle perturbe les catégories logiques avec lesquelles nous avons l'habitude de penser. C'est pourquoi toutes ces productions verbales procèdent $\mathrm{du}$ "piège " comme dit Breton dans Nadja. À côté de l'espace logique de la causalité surgit l'espace ouvert et béant du désir qui se cristallise en des images énigmatiques et sibyllines qui s'ouvrent à l'incertitude de l'interprétation. Dans ces images insolites, la rigueur de la logique qui distingue le sujet de l'objet s'effondre, les sujets et les objets s'entrecroisent et de leur mouvement ainsi que du bouleversement de leurs rapports, surgissent une temporalité et une spatialité nouvelles. L'éphémère et l'éternel se conjuguent dans une nouvelle temporalité dynamique, l'ici et l'ailleurs dans une nouvelle spatialité dynamique, ouvrant le champ au jeu libre de la perméabilité mutuelle des temps et des espaces ${ }^{6}$.

9 L'écriture automatique est en outre liée à la question de la représentation, posée par Breton sous les termes de la "perception primitive » et de sa recherche à travers la pratique poétique et picturale dans le but de bouleverser notre perception du réel. Liant directement peinture et littérature (BRETON, 1965, 357), Breton parle de 
déconstruction des habitudes perceptives, d'une ascèse du regard qui crée un voir toujours renouvelé à travers la décomposition et la recomposition continues de l'espace et des objets. La création poétique est liée à la peinture, car l'image et sa force de représentation concernent tant le voir que le dire. La subversion de la perceptionreprésentation nécessaire à l'émergence du désir concerne les deux arts dans une égale mesure. Engonopoulos est le surréaliste grec qui exerce parallèlement et de manière équivalente ces deux moyens d'expression artistique, invitant et provoquant à la fois le lecteur/spectateur à les appréhender comme un ensemble indissociable ${ }^{7}$. Le changement du regard porté sur les objets, les mots, les sujets, entraîne le changement du rapport dialectique entre intérieur/extérieur, moi/l'autre, objet/sujet, tant dans la poésie que dans la peinture. Le texte poétique devient aussi « spectacle » qui provoque admiration, surprise, embarras, trouble. Ainsi naît le merveilleux qui appelle à une libération de la perception lui permettant de mettre en question d'autres éléments de sorte à bouleverser les données et à laisser surgir de nouvelles significations. À travers un quasi «collage » d'images poétiques, Engonopoulos crée l'espace du surréel. Ses objets $^{8}$ et ses sujets ${ }^{9}$, dans les deux formes artistiques, sont porteurs de charges symboliques, ils deviennent des lieux énigmatiques et signifiants, car ils ne reproduisent pas les formes du monde extérieur, mais puisent dans la représentation intérieure (BRETON, 1962, 312). Il secoue ainsi les habitudes perceptives, dynamite les niaiseries du réalisme et laisse les portes grandes ouvertes au surréel.

10 Tout comme le tableau surprend, «regarde » et interpelle le sujet qui l'observe, le poème ne raconte pas, ne représente pas autant qu'il « fait voir » (LACAN, 1973, 98, 100). Les tableaux d'Engonopoulos nous invitent à lire la couleur, ses poèmes nous appellent à regarder le phonème (le signifiant). La réalité se révèle fragile devant la force de l'image dont l'intensité croît à la mesure de l'arbitraire qu'elle comporte. Selon Breton cet arbitraire procède entre autres des contradictions qu'elle contient, de la difficulté à la traduire en langage courant, de l'impression d'abstrait qu'elle confère au concret ou vice-versa (BRETON, 1979, 52-53). L'image, comme le verbe qui l'investit deviennent opaques, difficilement accessibles, suggérant le danger ou le risque. Le texte poétique reste ainsi «ouvert sur le vide comme une porte battante " $($ BRETON, 1978, 18) et à travers cette ouverture l'artiste laisse parler « l'autre prisme de vision » (BRETON, 1975).

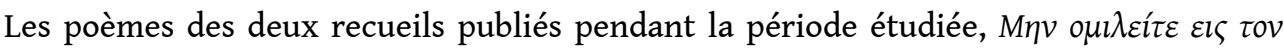

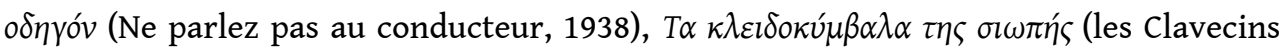

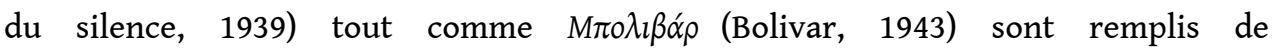
"surimpressions ", de nouvelles corrélations entre sujets et objets, de l'identification de la femme à l'inspiration poétique et artistique ${ }^{10}$, de références fréquentes à son art

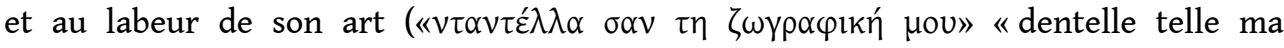
peinture ", $2^{\mathrm{e}}$ recueil, 126) ${ }^{11}$. Dans les corrélations bizarres, les enjambements de l'imagination, l'ironie subtile et l'humour noir ${ }^{12}$, le paradoxe des signifiants et des signifiés, l'imbrication de l'altérité du féminin avec l'inspiration et l'art ${ }^{13}$, Engonopoulos trouve les matériaux pour construire son art poétique.

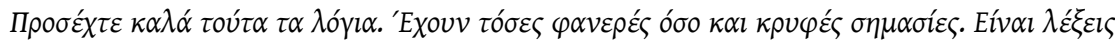

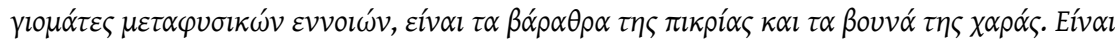

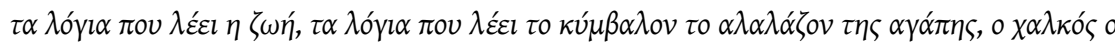

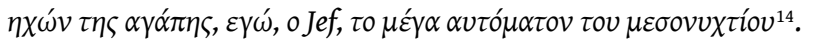
développer leurs sujets d'intérêt $t^{15}$, les valeurs nouvelles ${ }^{16}$ qu'ils veulent promouvoir, les techniques ${ }^{17}$ qu'ils utilisent, la construction d'une nouvelle tradition et d'une nouvelle 
identité artistique. Ils créent à travers l'écriture un univers de mots et d'images suscitant des transgressions constantes et répétées des interdits, des tabous, des censures, des canons littéraires et des canons des genres, pour inaugurer une nouvelle orientation du monde, de l'être et de l'art. Les productions rhétoriques et imaginaires tiennent lieu de prototypes de mondes à venir. Breton avait d'ailleurs déjà parlé du rapport entre l'imaginaire et ce que l'on se doit de vivre dans la réalité18. Pour les surréalistes, l'imaginaire constitue une fonction centrale du sujet à travers laquelle se développent la connaissance et l'action. Les surréalistes grecs se sont abandonnés à l'exaltation de l'imaginaire, persuadés que le réel n'est qu'une des formes possibles pour laquelle on renonce à toutes les autres. La poésie surréaliste agit contre cette renonciation.

La poésie d'A. Embiricos introduit comme éthique nouvelle le désir, parallèlement à la jouissance et au plaisir érotique ${ }^{19}$, où le corps retrouve son sens. La libération du désir implique le refus de le marginaliser, la recherche de sa satisfaction dans la rencontre vécue, dans l'événement subversif et dans le surréel. Le corps poétique, le corps des mots est la trace laissée sur son passage. L'amour est synonyme de l'expression du désir qui guide l'existence humaine même. Dans les deux premiers recueils poétiques

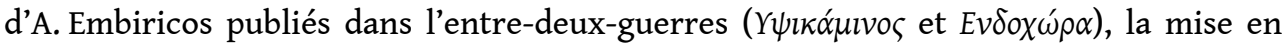
scène du désir est transportée dans le poème telle une force de mise en alerte constante. L'écriture poétique imite le désir dans son incessante mobilité et elle se comporte comme un corps érotique, de telle façon qu'effervescence poétique et érotique se confondent. "Le désir surgit au moment de s'incarner dans une parole " (LACAN, 1978, 273) et l'amour se manifeste dans la poésie embiricéenne tel un désir qui dure. D'ailleurs, pour A. Embiricos et les surréalistes, l'écriture automatique est une expression poétique issue de la racine du désir. L'amour est un foyer d'énergie, d'exaltation du désir et de volonté de vivre. L'idéalisation en forme une composante essentielle. La femme, intrinsèquement liée à l'amour ${ }^{20}$ devient le symbole du désir et, sous les multiples visages du désir, elle parcourt la scène poétique telle une perpétuelle présence/absence. Elle est aussi la "pierre angulaire du monde matériel » (BRETON,

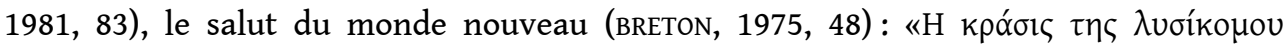

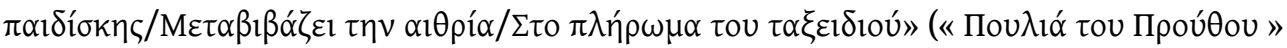

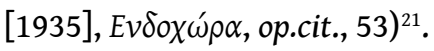

13 Pour Engonopoulos, le surréalisme est l'espace privilégié de la rencontre entre deux systèmes de signes auxquels il a accordé une grande importance: la langue et la peinture $^{22}$. En associant l'imaginaire à la mémoire, conformément à un modèle intérieur, il éveille la vie intérieure des objets, des sujets, des événements et des lieux, les abandonnant à la "fluctuation des rapports ${ }^{23}$ qui peuvent s'établir entre eux. Ainsi, le poème comme le tableau deviennent des foyers de significations multiples et des points de convergence de formes et de sens. Dans cette optique, le beau est relié au merveilleux et au mouvement ${ }^{24}$, effaçant toute différence entre métaphore poétique et picturale.

14 Pour les deux surréalistes grecs, la poésie est praxis et ne se réduit pas à la quête théorique de la vérité ou d'une version de la vérité. Toute revendication d'ordre moral, esthétique ou social se réalise à travers la pratique poétique. Pour outrepasser les

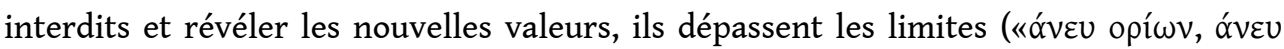
ó $\omega \omega v » ~ «$ sans conditions, sans restrictions », Embiricos) : du canon littéraire, du genre littéraire, de la pudibonderie, du lieu commun, du cloisonnement entre plaisir 
esthétique et érotique, entre imagination et réalité. «Tout relève de l'imagination et de l'imagination tout révèle » écrit ARAGON dans le Paysan de Paris (1982, 81). Leurs textes

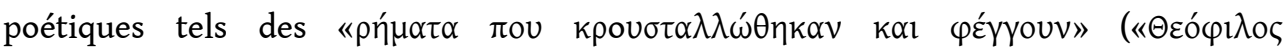

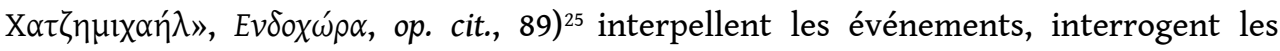
mœurs dominantes et annoncent, explicitement ou allusivement, la nouvelle éthique du désir.

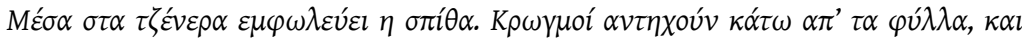

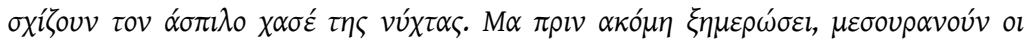

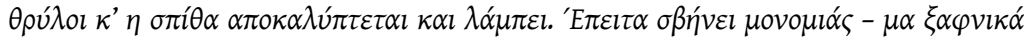

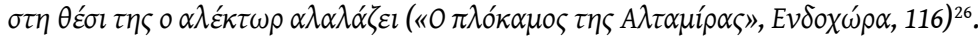

À travers les valeurs de la passion et de la subversion, ils présentent le réel par le biais d'une multitude de lentilles déformantes, afin de réexaminer le contenu du terme de réalité. De par la défamiliarisation que suscite chez les lecteurs de l'époque le paradoxe de leur discours poétique, ils proposent de nouveaux modèles d'écriture, un nouveau point de vue sur le monde, une nouvelle situation de l'être. L'art devient médiateur des valeurs de la vie et de la création, soulignant le rôle du sujet et lançant un regard différent sur les productions poétiques et artistiques du passé en cherchant à insuffler

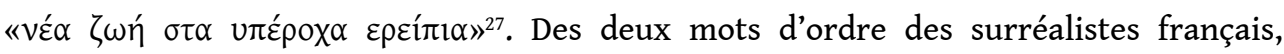
"transformer le monde» (emprunté de Marx) et "changer la vie» (emprunté de Rimbaud ${ }^{28}$, les surréalistes grecs n'ont tenté de mettre en œuvre que le second. L'absence en Grèce d'un groupe surréaliste cohésif auquel ils pourraient appartenir et se référer et qui leur permettrait de produire un discours théorique ${ }^{29}$ et critique et d'exprimer collectivement les libertés, les sensibilités et la créativité, a entravé la formation d'une élite ${ }^{30}$ de l'esprit qui aurait pu prétendre à construire une identité culturelle et littéraire nouvelle, apte à laisser des descendants. Ils ont cependant réussi à déployer, dans le présent atemporel du discours poétique, à travers leurs images insolites et étranges, une nouvelle façon de connaître et d'articuler le monde qui, dans le tourbillon de l'entre-deux-guerres (МАРАГК'ОПОҮАОг, 2002, 25-27), a malheureusement rencontré la non-lecture, la fausse lecture, le passage sous silence et la marginalisation.

\section{BIBLIOGRAPHIE}

ARAGON Louis, 1982, le Paysan de Paris, Paris : Gallimard/folio.

BRÉCHON Robert, 1971, le Surréalisme, Paris : Armand Colin.

BRETON André, 1982, l'Amour fou, Paris : Gallimard/folio.

BRETON André, 1981, les Vases communicants, Paris : Gallimard/idées.

BRETON André, 1979, Manifestes du surréalisme, Paris : Gallimard/idées.

BRETON André, 1978, Nadja, Paris : Gallimard/folio.

BRETON André, 1975, Arcane 17, Paris : J. J. Pauvert/10/18. 
BRETON André, 1965, le Surréalisme et la Peinture (1928), Paris : Gallimard.

BRETON André, 1962, Situation surréaliste de l'objet, Paris : J. J. Pauvert.

BRETON André, 1927, Introduction au discours sur le peu de réalité, Paris : Gallimard.

BRETON André et SOUPAULT Philippe, 1971, les Champs magnétiques (1919), Paris : Gallimard.

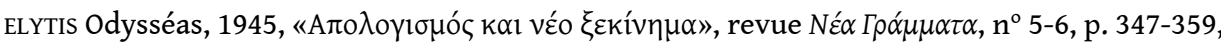

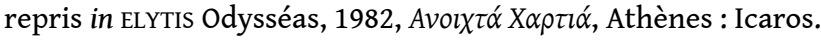

EMBIRICOS Andréas, 2001, Domaine intérieur, Trad. BoUchARD Jacques, Paris : L'Harmattan.

EMBIRICOS Andréas, 1991, « Des roses à la fenêtre », Haut Fourneau, Trad. BOUCHARD Jacques, Institut français d'Athènes, Avignon : Actes Sud.

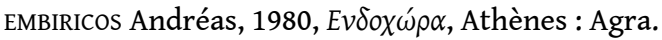

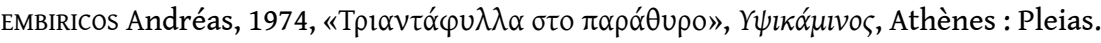

ENGONOPOULOS Nikos, 1985, Пoiń $\alpha \alpha \tau$, Tome II, Athènes : Icaros.

ENGONOPOULOS Nikos, 1977, Пoiń $\alpha \alpha \alpha$, Tome I, Athènes : Icaros.

LACAN Jacques, 1978, " Le désir, la vie et la mort ", le Séminaire, livre II, le Moi dans la théorie de Freud et dans la technique de la psychanalyse, Paris : Seuil.

LACAN Jacques, 1973, le Séminaire, livre XI, les Quatre concepts fondamentaux de la psychanalyse, Paris : Seuil.

LAUTRÉAMONT, 1969, Euvres complètes, Paris : Garnier/Flammarion.

Le Surréalisme ASDLR, 1931, $\mathrm{n}^{\circ}$ 3, décembre.

RIESE-HUBERT Renée, 1984, Du tableau-poème à la poésie concrète, Écritures II, Paris : Le Sycomore.

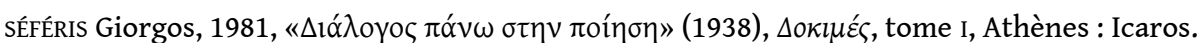

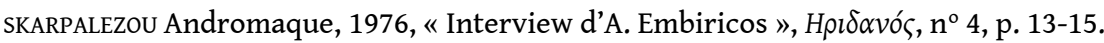

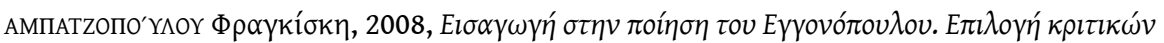

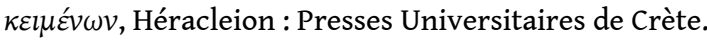

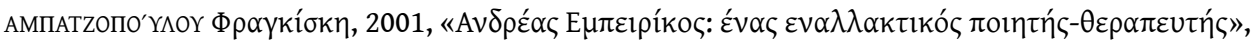

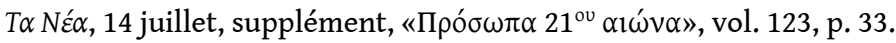

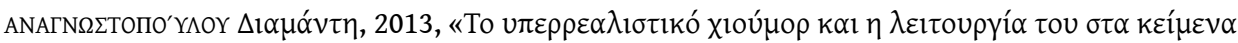

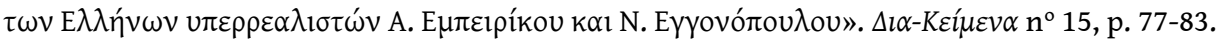
http://dia-Keimena.frl.auth.gr/index.php, consulté le 30 octobre 2015.

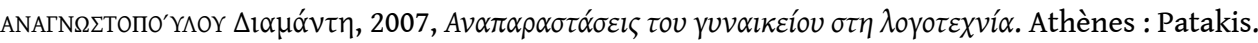

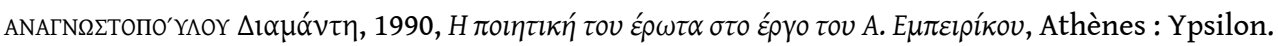

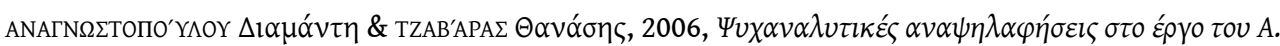

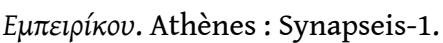

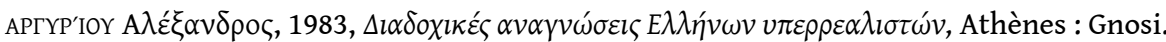

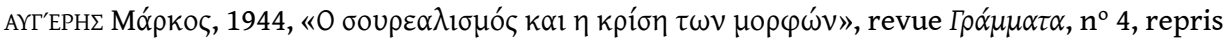
dans la revue $H \rho \imath \delta \alpha v o ́ \varsigma, ~ 4 / 1976$, p. 112-116. 


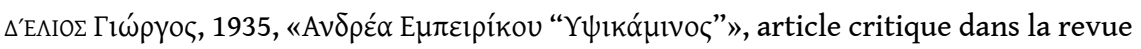

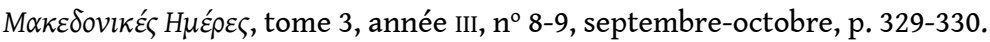

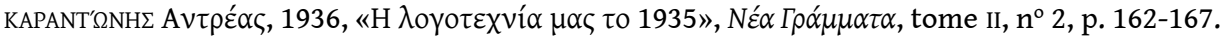

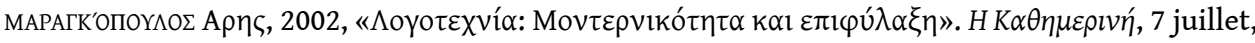

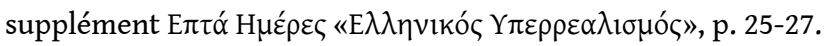

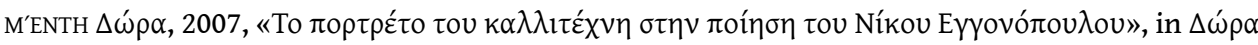

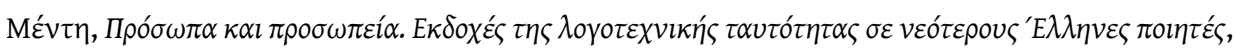
Athènes : Gutenberg, p. 115-177.

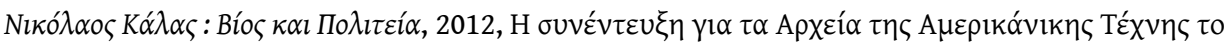

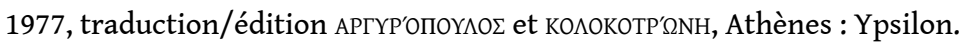

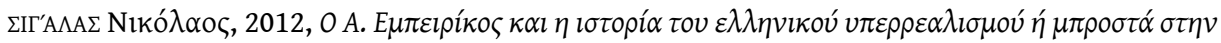

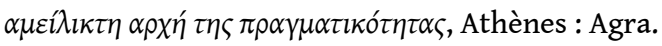

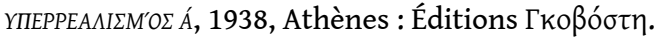

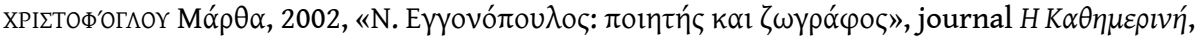

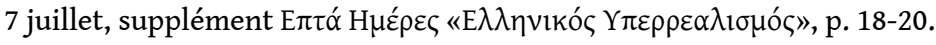

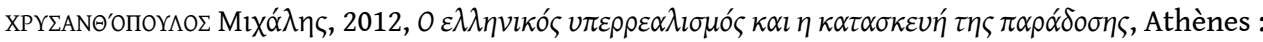
Agra.

\section{NOTES}

1. «Il existe des choses infiniment plus belles encore que cette sculpturale assiduité d'une épopée du passé » (A. EMBIRICOS, 1991, p. 53).

2. AYГ'ЕРНГ, M. 1944 et 1976, p. 112-116. Cf. aussi la réponse à ces critiques par ELYTIS, O. 1945, p. 347-359 et 1982, p. 378-394.

3. Cf. la note critique de quatre lignes d'A. Karantonis concernant $Y \psi \imath \kappa \alpha ́ \mu \imath v o \varsigma$ dont il dit : «alors qu'il pourrait servir de prétexte à un débat constructif sur le problème du surréalisme, il est devenu l'objet d'une ironie facile et inconsistante ", KAPANT'SNHE,

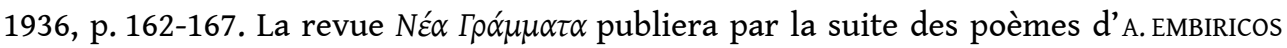
en mai 1937 (5), en 1938 (avril-mai 4-5) et en 1940 (1). Cf. également N. ENGonopoulos, 1977, notes p.146-150, 152, et l'interview d'A. Embiricos par A.Skarpalezou, en mars 1967 à Athènes, dans la revue $H \rho \imath \delta \alpha v o ́ s, 1976$, p. 13-15.

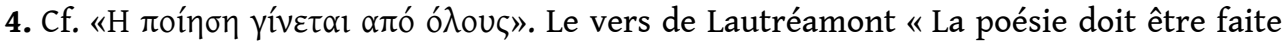
par tous. Non par un » (LAUTRÉAMONT, 1969, p. 291) devient le mot d'ordre de la première période (1919-1930) des surréalistes français.

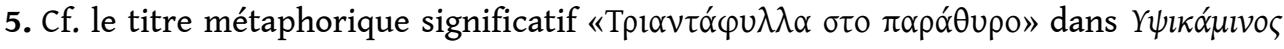
(EMBIRICOS, 1974, p. 49).

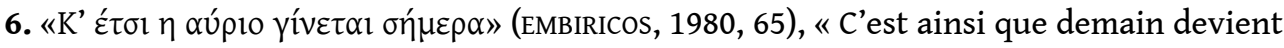
ce jour d'hui » (EMBIRICOS, 2001, p. 63).

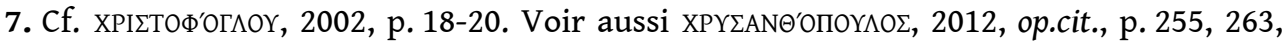

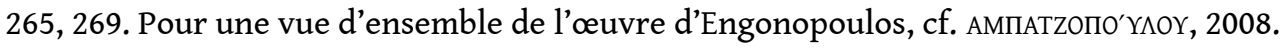


8. Machines à coudre, gramophones, tramway, marteaux, miroirs, harpes, clavecins, flûtes traversières, violons, phonographes, entonnoirs, statues, charrues, téléphones verts, ascenseurs, parapluies.

9. Bolivar, Eléonore, Guglielmo Zizzi, Androutsos, Robespierre, Ali Hadjar, Panaïs Koutalianos, Rigas Feraios, Oikonomou, Isidore Ducasse, etc.

10. Par exemple, $1^{\text {er }}$ recueil, p. 22, 30, 157, $2^{\mathrm{e}}$ recueil, p. 113, 126.

11. Cf. M'ENTH, 2007, p. 115-177.

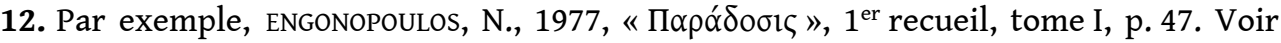

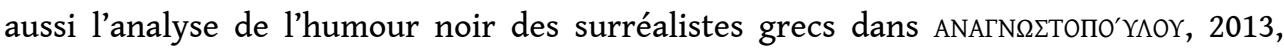
p. 77-83.

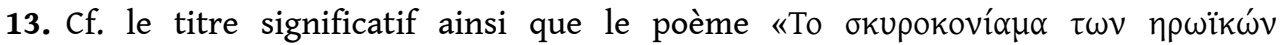
$\pi \alpha \rho \theta \varepsilon ́ v \omega v »$ [Le béton des vierges héroïques], $2^{\mathrm{e}}$ recueil, p. 113.

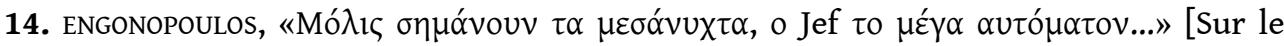
coup de minuit, Jef, le grand automate...], $2^{\mathrm{e}}$ recueil, p. 133-134. ( Faites bien attention à ces mots. Ils ont autant de sens apparents que cachés. Ce sont des mots pleins de significations métaphysiques, ce sont les gouffres de l'amertume et les sommets de la joie. Ce sont les mots dits par la vie, les mots dits par la cymbale retentissante de l'amour, le cuivre résonnant de l'amour, moi, Jef, le grand automate de minuit »- notre traduction).

15. Le monde d'hier et de demain, le développement de la sexualité, la femme-nature et la femme-vision, l'inspiration artistique, l'érotisme, la langue nouvelle et l'art nouveau, la proclamation d'une existence nouvelle, d'une vie nouvelle, le désir et l'importance de l'inconscient.

16. La quête de l'amour, de la poésie, de la liberté.

17. Automatisme, substitution métaphorique, libre association, jeux de mots, émergence de l'a-logique et du paradoxal, le rêve, le merveilleux, la "surimpression ", le « poème-événement ».

18. «[...] la conversion de plus en plus nécessaire [...] de l'imaginé au vécu ou plus exactement au devoir-vivre » (BRETON, 1981, p. 10-11).

19. Cf. АNAГN $\Omega E T O \Pi O^{\prime} Y \Lambda O Y, 1990$, et 2007, p. 253-300, ainsi que ANAГN $\Omega E T O \Pi O^{\prime} \Upsilon \Lambda O Y$ et TZAB'APAE, 2006, p. 15-76.

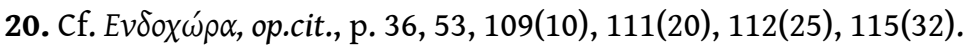

21. "La frimousse de la gamine à la flottante chevelure/Communique à l'équipage de la croisière/La sérénité de l'azur » EMBIRICos, «Les oiseaux de la Pruth» in Domaine intérieur, op. cit., p. 52.

22. Cf. à ce sujet l'analyse approfondie de Renée RIESE-HUBERT, 1984. Voir également les

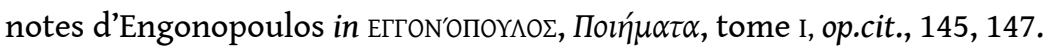

23. BRETON, A. le Surréalisme et la Peinture, op.cit., 269-270. Pendant les années 1920 et 1930, il se produit dans le milieu surréaliste une réflexion sur les «objets surréalistes »; cf. BRETON, A. Introduction au discours sur le peu de réalité (1927) et Le Surréalisme ASDLR, nº 3, déc. 1931. À peu près à la même période (1941) Breton invente le " poème-objet » qui associe les sources de la poésie et de la peinture.

24. «La beauté convulsive sera érotique-voilée, explosante-fixe, magiquecirconstantielle ou ne sera pas » (BRETON, 1982, p. 26). 
25. « À peine cristallisé le verbe scintille », in Domaine intérieur, op. cit., p. 84.

26. «L'étincelle est enfouie parmi les cendres. Des croassements retentissent sous la feuillée et déchirent de la nuit la toile immaculée. Mais avant même qu'il fasse jour, les rumeurs culminent et l'étincelle se révèle sublime. Puis soudain elle s'éteint - mais à sa place aussitôt le coq s'égosille» («La natte d'Altamira » in Domaine intérieur, op. cit., p. 105).

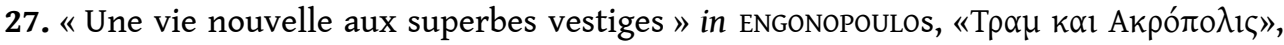

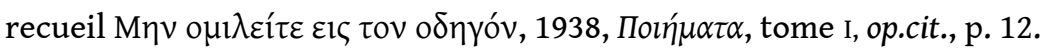

28. Cf. A. BRETON, Discours au Congrès des écrivains, Position politique du surréalisme (1935), p. 285.

29. Seul N. Kalas s'est efforcé d'articuler une réflexion théorique, à travers ses articles dans les revues de l'époque et dans sa tentative de présenter le terme, la définition et

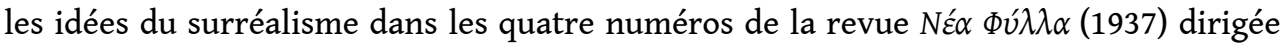

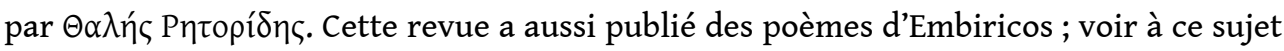

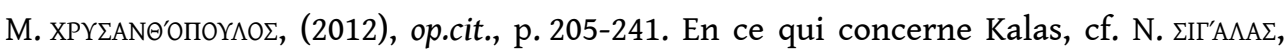

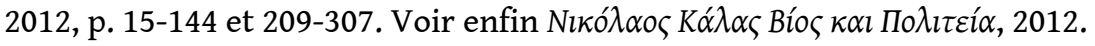

30. Le terme d'élite revêt souvent une connotation négative et appelle à la circonspection. Ce qui est mis en cause est l'idée qu'une supériorité, fût-elle considérée positivement, puisse conférer des droits différents, c'est-à-dire de supériorité, à ceux qui n'appartiennent pas à cette élite. Les surréalistes parlaient d'ailleurs de la "poésie faite par tous", de "la poésie [qui] est dans la rue ». Comment les imaginer alors comme les représentants d'une élite ou de l'élitisme? Mais même dans le sens positif du terme, c'est-à-dire comme des individus qui auraient adopté une position dominante pour réformer l'identité culturelle de leur pays, les surréalistes grecs n'appartiennent pas à cette catégorie: cela présuppose d'une part l'appartenance à un groupe dans lequel se développeraient les concepts nouveaux et d'autre part qu'ils aient soutenu et revendiqué dans la sphère publique une réflexion théorique complète. À la même

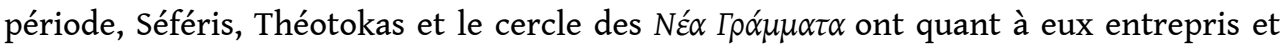
réalisé une action de ce type.

\section{RÉSUMÉS}

La conception surréaliste met en scène poétique les forces agissantes du désir, vers la quête de la connaissance approfondie de l'Autre et de la conciliation du champ intérieur et du champ extérieur.

Il gît toujours dans l'écriture un mouvement du désir qui résulte de deux altérités : l'interdit venant des autres, de la loi extérieure, et la force de la pulsion venant de l'intérieur. Il y a alors deux contraintes à transgresser, d'une part la loi et, d'autre part, la contrainte endogène, psychique. Le surréalisme s'est efforcé à sa façon, de concilier des entre-deux : un entre-deux réalités, un entre-deux espaces, un entre-deux sexes. La littérature devient l'exploration de l'entre-deux et écrire s'affirme pour les surréalistes comme une façon de penser cet entre-deux. La force de transgression, inhérente au champ surréaliste révèle la permanence de l'amour 
comme constructeur des espaces de paroles poétiques. À travers cette communication, on explorera la façon dont les surréalistes grecs (et surtout Embiricos et Engonopoulos) ont assumé le rôle d'élite dans la formation d'une nouvelle identité culturelle de leur temps, la façon dont ils se sont mesurés aux interdits, aux conflits et à la transgression et les représentations de la coupure et de la rupture avec le passé dans leur œuvre.

The surrealist conception puts forward on the poetical scene the driving forces of desire, in quest of the deeper knowledge of the "Other" and of the conciliation of the interior and exterior fields. Writing is always underlain by a movement of desire resulting from two alterities: the taboo originating from the others, from the exterior law, and the force of the drive stemming from inside. Two kinds of constraints must then be broken: those of law, and the endogenous, psychic ones. Surrealism attempted in its own way to reconcile in-betweens: an in-between realities, an in-between spaces, an in-between sexes. Literature becomes the exploration of in-betweens and writing asserts itself as a way to think this in-betweenness. The force of transgression inherent to the surrealist field reveals the permanence of love as a builder of spaces for poetical words. This paper aims to explore the way in which Greek surrealists (and mainly Embirikos and Engonopoulos) assumed the role of an elite in the formation of a new cultural identity of their time, the way in which they have measured themselves against taboos, conflicts and transgression and the representations of cleavage and break with the past in their work.

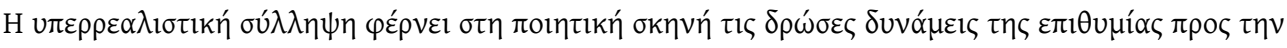

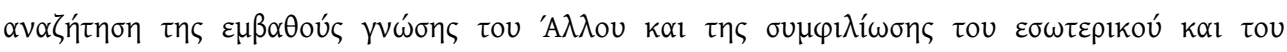

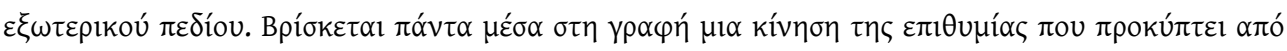

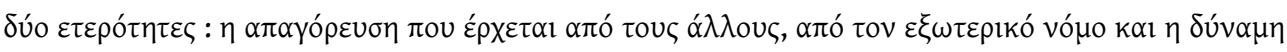

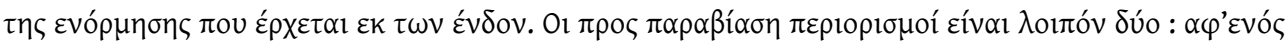

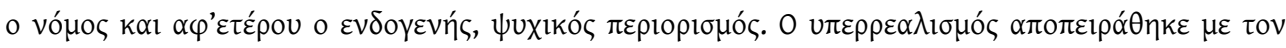

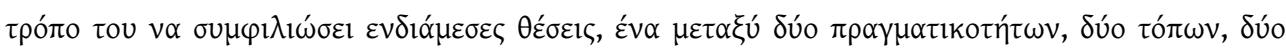

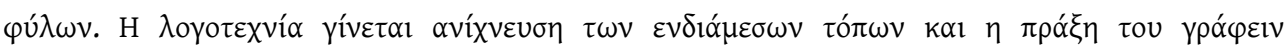

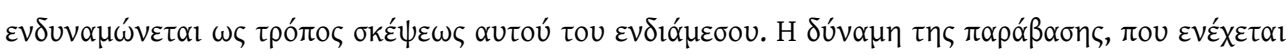

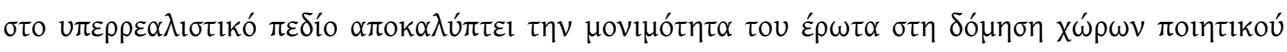

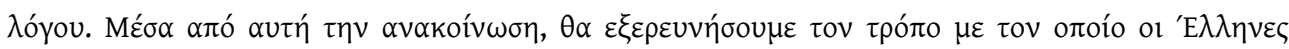

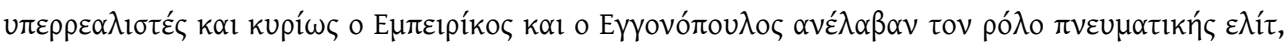

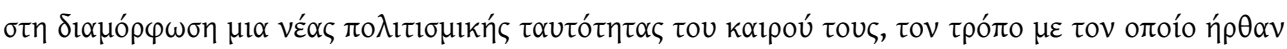

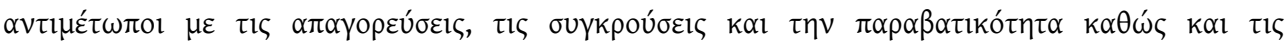

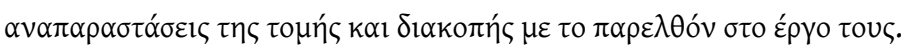

\section{INDEX}

Index géographique : Grèce

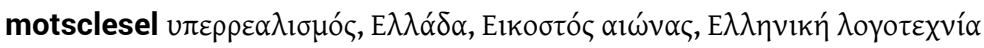

motsclestr Gerçeküstücülük, Yunanistan, Yirminci yüzyıl, Yunan edebiyatı

motsclesmk НАДРЕАЛИЗАМ, ГРЦИЈА, ДВАЕСЕТТИОТ ВЕК, ГРЧКАТА ЛИТЕРАТУРА

Keywords : surrealism, Greece, Twentieth century, Greek literature

Mots-clés : surréalisme, Politis Nikolaos (1852-1921)

Thèmes : Littérature grecque

Index chronologique : vingtième siècle 
AUTEUR

DIAMANTI ANAGNOSTOPOULOU

Université de l'Égée 